\title{
Blistering Diseases in A Tertiary Care Hospital: A 10-year Audit
}

\author{
Parajuli $\mathbf{N}^{1}$, Lama L ${ }^{1}$, Karki $\mathbf{A}^{1}$, Shrestha $\mathbf{R}^{1}$, Tiwari $\mathbf{S}^{2}$, Kayastha $\mathbf{B M M}^{3}$ \\ ${ }^{1}$ Department of Dermatology and Venereology, ${ }^{2}$ Department of Pathology, National Academy of Medical Sciences, \\ Kathmandu, Nepal, ${ }^{3}$ Department of Dermatology and Venereology, Patan Academy of Health Sciences, Patan, Nepal
}

\begin{abstract}
Introduction: Cutaneous blisters can occur in variety of conditions. They are broadly classified as immunobullous or non-immunobullous. Immunobullous blistering diseases can further classify as intraepidermal or subepidermal. Nonimmunobullous blistering disease are commonly due to infections, drugs among others. Some of the blistering disease carry significant mortality and morbidity. There are only limited data on blistering diseases from Nepal, so this study will provide details on the prevalence of this rare dermatological disease from one of the tertiary care referral hospital in the country.

Objective: To assess the clinical pattern and prevalence of various blistering diseases in a tertiary care referral hospital. Materials and Methods: All of the data were obtained from the admission register maintained at department of dermatology and venereology, National Academy of Medical Sciences. All the patients admitted with blistering disease from year April 2008 till March 2018 were included. The details including age, sex, type of blistering disease and length of hospital stay were recorded.

Results: A total of 710 patients were admitted in the dermatology ward over the period of 10 years. There were a total of 193 blistering disease admitted during this period. Autoimmune blistering disease was diagnosed in 59 patients whereas 100 patients had non-immunobullous disease. The age grouped range from 4- 93 years with the mean age of $43 \pm 19.71$ years. The average number of hospital stay of all blistering diseases was $11.73 \pm 11.36$ days.

Conclusion: Blistering diseases are one of the most common conditions requiring admission in dermatology ward. Some of these diseases carry a significant morbidity and mortality. So, prompt diagnosis and treatment is of utmost importance.
\end{abstract}

Key words: Autoantibodies; Fluorescent Antibody Technique; Pemphigoid; Bullous; Pemphigus; Skin Diseases; Vesiculobullous

\section{Introduction}

Ceveral dermatological diseases are characterized by the formation of blisters. Blisters can either be acellular or filled with particular inflammatory cells. ${ }^{1}$ Cutaneous blisters occur in a wide variety of clinical settings, including autoimmune disorders, drug reactions, infections, genetic disorders, and physical injury. ${ }^{2}$

Financial disclosure: None.

Conflict of interest to disclosure: None declared.

Address of Correspondence

Dr. Niraj Parajuli

Assistant Professor

Department of Dermatology

National Academy of Medical Sciences

Kathmandu, Nepal.

E-mail:drnirajparajuli@gmail.com
Blistering diseases are a heterogeneous group of disorders that can affect either skin and mucous membrane, or both, varying in presentation, clinical course, histology, immunopathology and treatment. Few of blistering diseases are severe in nature,

Submitted: $10^{\text {th }}$ October 2019

Accepted: $30^{\text {th }}$ December 2019

Published: $7^{\text {th }}$ October 2020

\section{How to cite this article}

Parajuli N, Lama L, Karki A, Shrestha R, Tiwari S, Kayastha BMM. Blistering diseases in a tertiary care hospital: A 10-year audit. Nepal Journal of Dermatology Venereology and Leprology 2020;18(1):24-7. https://doi.org/10.3126/njdvl.v18i1.30259.

\section{(c) (7)}

Licensed under CC BY 4.0 International License which permits use, distribution and reproduction in any medium, provided the original work is properly cited. 
and sometimes fatal. ${ }^{3}$ So, it is essential to be able to recognize the severe life-threatening blistering conditions and provide appropriate treatment and management. $^{2}$

Blistering of the skin can be broadly classified as either immunobullous (autoimmune) or nonimmunobullous.

Autoimmune blistering diseases can be categorized into two groups: intraepidermal and subepidermal. Intraepidermal or pemphigus diseases is characterized by intraepidermal blistering and autoantibodies against desmosomal proteins such as desmoglein, members of the plakin family. Subepidermal blistering diseases, which are comprised of pemphigoid diseases and dermatitis herpetiformis. ${ }^{4}$

Non-immunobullous causes of blistering diseases includes infections, drugs and other rare metabolic disorders. $^{5}$

Clinical pattern of the blister can suggest the etiology but a proper diagnosis usually require a biopsy for histopathology examination along with some special tests including immunofluorescence and serological assays. ${ }^{5}$ There are only few published studies on blistering diseases from Nepal to the best of our knowledge. Hence, this study was done to assess the clinical pattern and prevalence of various blistering diseases in a tertiary care referral hospital of Nepal. This study will pave way for further studies in this form of dermatological disorders.

\section{Materials and Methods}

A retrospective audit of all the patients admitted in dermatology ward of National Academy of Medical Sciences, Bir Hospital, Kathmandu, Nepal with presence of blisters as a chief complaint during admission were included. An ethical clearance was obtained from the institutional review board of National Academy of Medical Sciences, Bir Hospital before embarking on the study.

A detailed data from the admission records of all cases admitted in dermatology ward from April 2008 to March 2018 for a total of 10 years were recorded. All the demographic details like age, sex, address and number of days admitted were included in this study. All the data were analyzed using the version of SPSS 22.

\section{Results}

Record of the admitted patients showed that a total of 710 patients were admitted in the dermatology ward over the period of 10 years. A total of $193(27.18 \%)$ blistering diseases were admitted, among whom 159 (22.39\%) were new cases and 34 patients were repeat admissions. Among the total admitted cases, autoimmune blistering diseases were diagnosed in 59 (8.3\%) patients whereas $100(14.08 \%)$ patients had non-immunobullous disease. Some of the patients with autoimmune forms of blistering disease were admitted multiple times over the course. The age of blistering diseases ranged from 4-93 years with the mean age of $43 \pm 19.71$ years. The blistering diseases were more common in older age groups (more than 50 years).

The maximum number of total dermatological admissions in a single year was 116 new cases in year 2011/12 with 28 new cases of blistering disease, which included nine cases of autoimmune blistering diseases. The lowest number of admission was of only 41 patients in year 2014/15 with nine cases of blistering disease, among which four cases were of autoimmune blistering disease (Figure 1).

Among the total admission, there was a total of 98 males (50.8\%) and 95 females (49.2\%). Similarly, the distribution of admission according to the immunological classification, autoimmune blistering diseases constituted 71 admissions (37\%) only as compared to 122 (67\%) admissions of nonimmunological blistering diseases

The most common causes of blistering diseases were medications (30\%) including the epidermal necrolysis. The other common causes included herpes virus infection $(22.6 \%)$, followed by pemphigus vulgaris (19.5\%).

In general, the autoimmune blistering diseases constituted only $8.3 \%$ of the total admission. Among the autoimmune disease, there were a total of 31 pemphigus vulgaris (22.6\%), 13 pemphigus foliaceus $(8 \%)$ and 10 bullous pemphigoid $(6.3 \%)$ which is depicted in the figure below (Figure 2).

The average length of hospital stay for blistering diseases was $11.73 \pm 11.36$ days. 


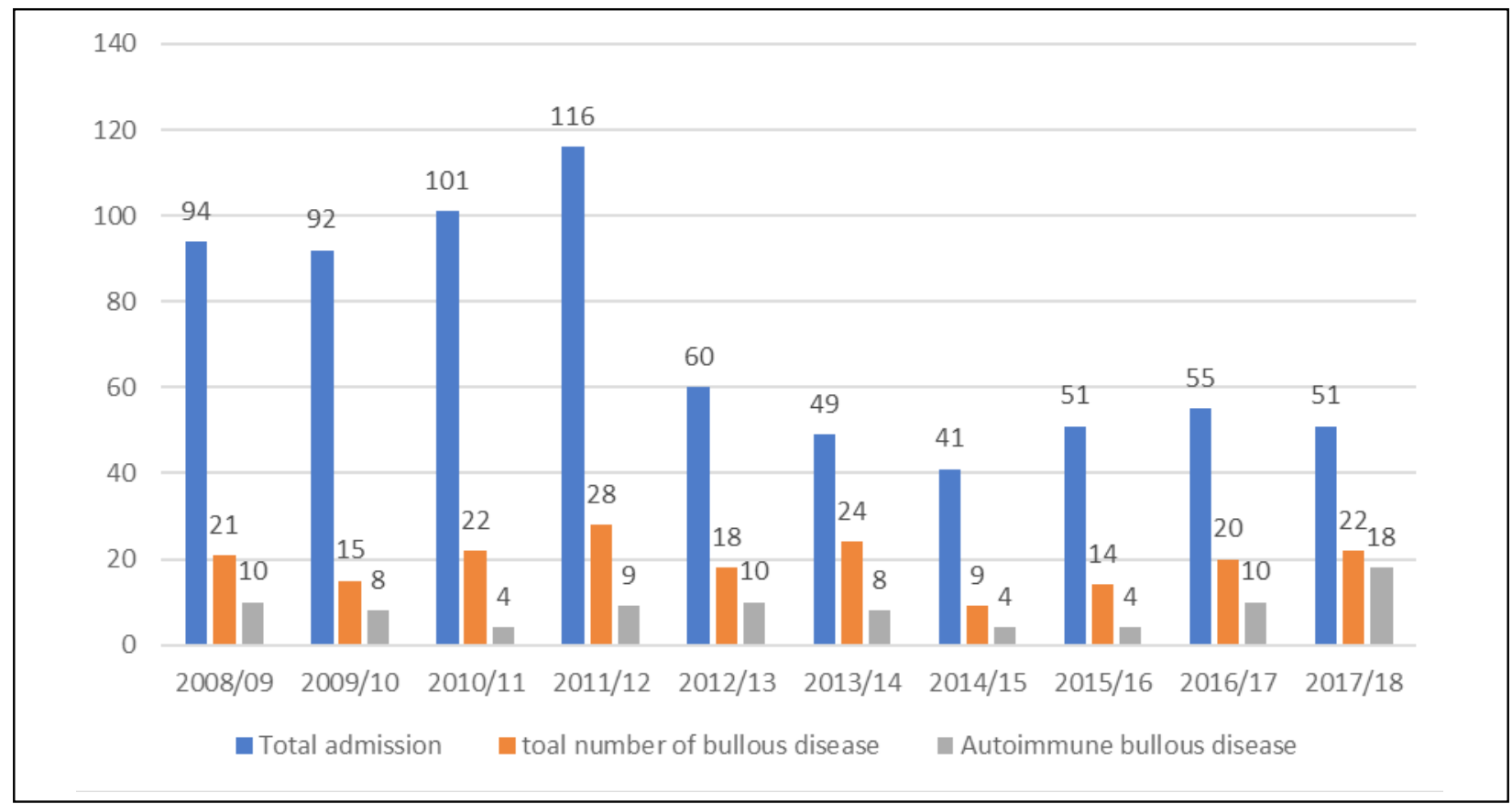

Figure 1: Bar diagram showing the distribution of total admission, bullous diseases and autoimmune blistering disease over the 10-year period.

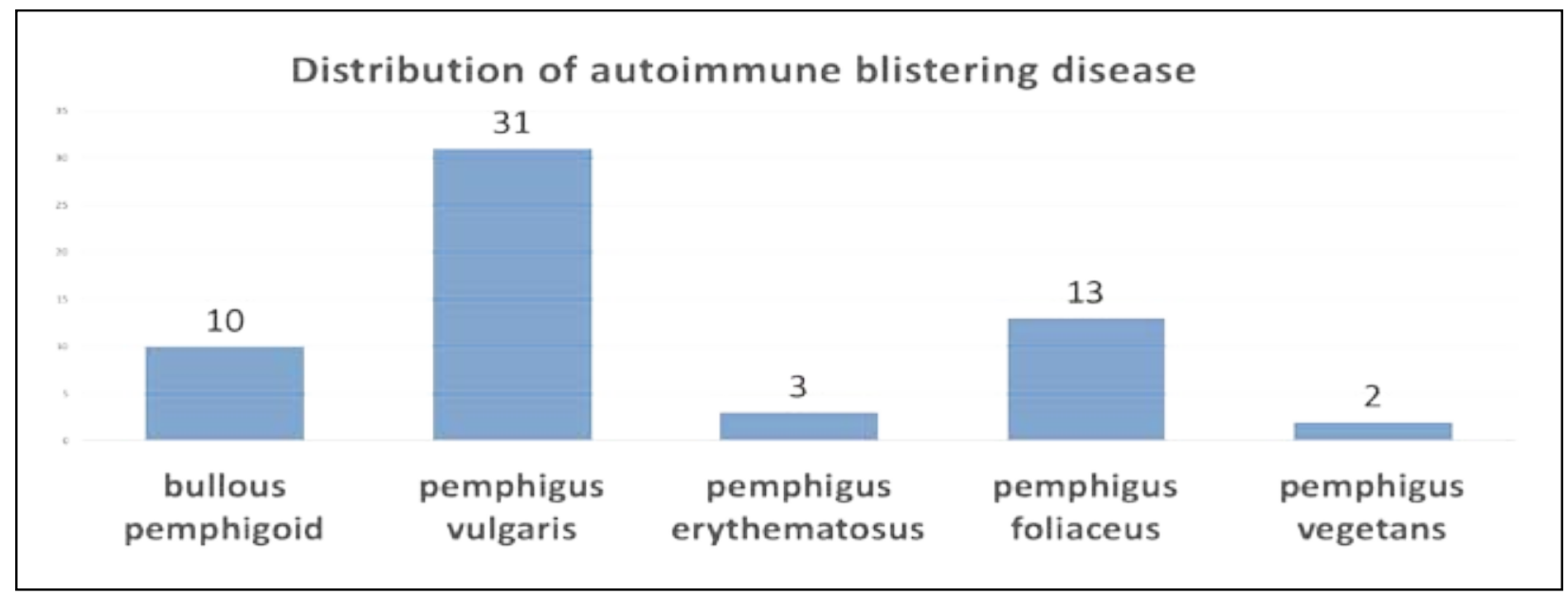

Figure 2: Bar graph showing the distribution of autoimmune blistering disease for the 10-year period.

\section{Discussion}

Blistering of the skin can be due to a number of diverse etiologies. Some blistering eruptions are self-limited but others are life-threatening which requires prompt diagnosis and management. ${ }^{6}$ Infections and drug eruptions constituted the most common diagnosis in skin ward which was the same in our study too. ${ }^{5}$ Our study showed that almost two third (63\%) were nonimmunobullous in nature and only $37 \%$ of the total cases were autoimmune among the admitted cases.

Studies from hospitals in Nepal and India have shown that the blistering diseases were one of the common conditions requiring admission in dermatology ward. ${ }^{7,8}$ Similarly, our study showed that out of the 710 total admission over the 10 years period, $193(27.18 \%)$ were of blistering diseases.

One of the major causes of mortality among dermatological admission is from epidermal necrolysis. A study from United States found that mean adjusted mortality was in an upward trend from $4.8 \%$ for SJS, $19.4 \%$ for SIS/TEN to $14.8 \%$ for TEN. ${ }^{9}$ Our study had two mortalities and both were secondary to epidermal 
necrolysis. The mean duration of hospital stay in our study was shorter as compared to another study from Nepal ${ }^{8}$ but longer than that from India. ${ }^{7}$ The shorter duration in study from Kathmandu is probably due to the fact that many of their patients were admitted just to receive the pulse medications.

Few studies have shown that patients with autoimmune blistering disease have a higher mortality as compared to general population. ${ }^{10,11}$ But, our study failed to show such associations. Despite the rarity of autoimmune blistering diseases physicians should be aware of autoimmune blistering diseases which carry a significant morbidity and mortality. ${ }^{5}$

All cases were diagnosed by clinical and histopathological examination only, except in two cases which underwent direct immunofluorescence

\section{References}

1. Wick MR. Bullous, pseudobullous, \& pustular dermatoses. Semin Diagn Pathol 2017 (May);34(3):250-60. https://doi.org/10.1053/j. semdp.2016.12.001

2. David W. The vesicobullous reaction pattern. 3rd ed. Edinburgh: Elsevier; 2010. p123

3. Yeh SW, Ahmed B, Sami N, Razzaque Ahmed A. Blistering disorders: diagnosis and treatment. Dermatol Ther 2003;16(3):214-23. https://doi. org/10.1046/j.1529-8019.2003.01631.x

4. Van Beek N, Zillikens D, Schmidt E. Diagnosis of autoimmune bullous diseases. JDDG: Journal der Deutschen Dermatologischen Gesellschaft 2018 (Sep);16(9):1077-91. https://doi.org/10.1111/ ddg.13637

5. Welsh B. Blistering skin conditions. Aust Fam Physician 2009 (Jul);38(7):484-90.

6. Jaleel $T$, Kwak $Y$, Sami N. Clinical Approach to Diffuse Blisters. Med Clin North Am 2015 (Nov);99(6):1243-67, xii. https://doi. org/10.1016/j.mcna.2015.07.009

7. Sen A, Chowdhury S, Poddar I, Bandyopadhyay D. Inpatient dermatology: Characteristics of patients and admissions in a tertiary level examination. However, our study failed to include cases of blistering diseases from the dermatology outpatient department.

\section{Conclusion}

This study showed the prevalence of blistering diseases among the admitted cases from one of the tertiary care centers. Blistering diseases are one of the most common conditions requiring admission in the dermatology ward. Some of these diseases carry a significant morbidity and mortality. Prompt recognition and treatment is the key in decreasing the mortality as well as morbidity associated with these rare skin conditions. Further larger studies including patients from skin out-patient departments with blistering disease would further enhance on the detail epidemiology of these disorders. hospital in Eastern India. Indian J Dermatol 2016 (Sep);61(5):561. https://doi.org/10.4103/00195154.190104

8. Parajuli S, Paudel U, Pokhrel DB. In patient dermatology: characteristics of patients and admissions in Tribhuvan University (TU) Teaching Hospital. Nepal Journal of Dermatology Venereology and Leprology 2013 (Apr);11(1):5963. https://doi.org/10.3126/njdvl.v11i1.7936

9. Hsu DY, Brieva J, Silverberg NB, Silverberg JI. Morbidity and mortality of Stevens-Johnson syndrome and toxic epidermal necrolysis in United States adults. Journal of Investigative Dermatology $2016 \quad$ (Jul);136(7):1387-97. https://doi.org/10.1016/j.jid.2016.03.023

10. Huang $\mathrm{YH}$, Kuo CF, Chen YH, Yang YW. Incidence, mortality, and causes of death of patients with pemphigus in Taiwan: a nationwide population-based study. J Invest Dermatol 2012 (Jan);132(1):92-7. https://doi.org/10.1038/jid.2011.249

11. Roujeau JC, Lok C, Bastuji-Garin S, Mhalla S, Enginger $V$, Bernard P. High risk of death in elderly patients with extensive bullous pemphigoid. Archives of dermatology 1998(Apr);134(4):465-9. https://doi.org/10.1001/archderm.134.4.465 